\title{
Escenarios de exposición a monóxido de carbono que orientan la sospecha clínica de intoxicación aguda
}

Carbon monoxide exposure scenarios that may guide a clinical suspicion of severe poisoning

Cenários de exposição ao monóxido de carbono que orientam a suspeita clínica de envenenamento agudo

Dras. María Noel Tortorella', Amalia Laborde²

\section{Resumen}

El monóxido de carbono (CO) se acumula en ambientes interiores cuando hay combustión y no es perceptible. Los síntomas de intoxicación son inespecíficos, por lo que las circunstancias en las que ocurre la intoxicación pueden ser orientadoras para el diagnóstico.

Objetivo: fortalecer capacidades clínicas para sospechar y confirmar intoxicaciones por CO.

Método: estudio descriptivo transversal de los casos sospechosos (pacientes con síntomas neurológicos o digestivos en los que se identifica una fuente de combustión en ambiente interior) consultados en el Centro de Información y Asesoramiento Toxicológico en 2017.

Resultados: fueron 111 casos, de los cuales 52 ocurrieron en 22 episodios de exposición colectiva. Los menores de 15 años fueron $37 / 111$ y el porcentaje de niños que se identificó en intoxicaciones colectivas (23/37) es significativamente mayor que en adultos. En el período invernal ocurrieron $67 / 111$, siendo la temperatura mínima media de $9,3^{\circ} \mathrm{C}$. Los equipos a combustión involucrados con mayor frecuencia fueron los calefones a gas, seguidos de las estufas a gas. El síntoma inicial más frecuente fue la cefalea. La media de la carboxihemoglobina (COHb) fue de 14,7\%. Los casos severos (45/111) se correlacionaron significativamente con la exposición a $\mathrm{CO}$ de un calefón a gas.

Conclusiones: la tasa de intoxicación es baja comparada con países de similar latitud, lo que plantea la posibilidad de un subdiagnóstico. La temperatura mínima ambiental por debajo de $10^{\circ} \mathrm{C}$, la permanencia en espacios con calefacción a combustión y el uso de calefón a gas fueron los escenarios típicos de la intoxicación. La cefalea es un síntoma clave para investigar la exposición. La sospecha diagnóstica puede ser menor cuando se trata de casos individuales, sobre todo en niños.

Palabras clave: Monóxido de carbono

Intoxicación por monóxido de carbono

Key words: $\quad$ Carbon monoxid

Carbon monoxide poisoning

1. Profesora adjunta del Departamento de Toxicología, Hospital de Clínicas, Facultad de Medicina, Universidad de la República.

2. Profesora del Departamento de Toxicología, Hospital de Clínicas, Facultad de Medicina, Universidad de la República.

Correspondencia: Dra. María Noel Tortorella. Correo electrónico: marianoeltortorella@gmail.com

Aprobado por el Comité de Ética del Hospital de Clínicas.

Las autoras declaran no tener conflictos de intereses.

Recibido: $26 / 8 / 2020$

Aprobado: 13/1/2021

Attribution-NonCommercial 4.0 International (CC BY-NC 4.0) 


\section{Introducción}

La intoxicación por monóxido de carbono (CO) presenta varios desafíos diagnósticos. Desde las décadas de 1970 y 1980 hasta la actualidad múltiples artículos científicos utilizan términos como enfermedad "oculta", causada por un tóxico "silencioso", que induce síntomas "camaleónicos", o como un gran "imitador" de enfermedades. Esto es debido a dos razones principales: la inespecificidad de los síntomas que admiten otros diagnósticos de patologías frecuentes y la falta de noción de exposición porque es un gas imperceptible por los senti$\operatorname{dos}^{(1-3)}$.

El gas no se ve ni se huele, y no provoca síntomas irritativos, por lo que puede inhalarse durante horas sin ser percibido. El CO se genera cuando hay combustión independientemente de la presencia de humo. El mecanismo de acción tóxica del $\mathrm{CO}$ es la asfixia celular de origen químico, por unión con la hemoglobina y otras globinas, y por inhibición de las citocromo $\mathrm{C}$ oxidasas mitocondriales. A la hipoxia celular se asocia un mecanismo de estrés oxidativo y un proceso inflamatorio generalizado ${ }^{(1)}$.

La intoxicación aguda por $\mathrm{CO}$ se manifiesta con síntomas como cefaleas, náuseas, fatiga, debilidad, lipotimia o síncope. Estos síntomas orientan diversos diagnósticos diferenciales: cuadros virales, cefaleas tensionales, cuadros de origen gastrointestinal, neurológico o cardiovascular. Síntomas de mayor gravedad: coma y convulsiones o infarto agudo de miocardio plantean como diagnóstico diferencial la encefalitis, accidente vascular, o enfermedad isquémica cardíaca ${ }^{(3,4)}$.

La confirmación diagnóstica se realiza mediante la dosificación de la carboxihemoglobina $(\mathrm{COHb})$, aunque su valor es dependiente de la oportunidad de la toma de muestra con respecto al tiempo trascurrido desde la exposición y la administración previa de oxígeno $\left(\mathrm{O}_{2}\right)$ ${ }^{(5,6)}$. El valor normal de $\mathrm{COHb}$ es de $2 \%$ y en los fumadores la $\mathrm{COHb}$ puede ser basalmente cercana a $10 \%{ }^{(4)}$.

La intoxicación por $\mathrm{CO}$ puede ser fatal y quienes sobreviven pueden tener secuelas neurológicas y cardiovasculares. Se reporta un aumento de la mortalidad por cualquier causa y un aumento de la incidencia de infartos de miocardio en pacientes que han sufrido esta intoxicación ${ }^{(7,8)}$. Síntomas neurológicos secuelares, por ejemplo, pérdida de concentración, de memoria y alteraciones del carácter, se describen en hasta un $40 \%$ de los pacientes que sufrieron intoxicaciones agudas. No se conoce con certeza el porcentaje de otras secuelas neurológicas, como síndrome parkinsoniano, coreatetosis, demencia o alteraciones cardiovasculares ${ }^{(9,10)}$.

La evidencia sugiere que muchas intoxicaciones por CO no son diagnosticadas. La falta de identificación de estos casos evita que se tomen medidas de prevención y favorece la exposición reiterada, continua o intermitente, de la misma u otras personas y, con ello, el aumento del riesgo de sufrir nuevos episodios agudos o daños neurológicos, o cardiovasculares crónicos ${ }^{(3,11)}$.

Se reconoce que la intoxicación por $\mathrm{CO}$ ocurre mayormente en espacios interiores, frecuentemente en el hogar, en circunstancias en las que se utilizan equipamientos o procesos a combustión (a gas, carbón, leña y otros) para calefaccionar ambientes, para disponer de agua caliente o para cocinar ${ }^{(12,13)}$.

No percibir su presencia lleva a la falta de noción de contacto y la inespecificidad de los síntomas hace improbable la sospecha clínica. El estudio realizado apunta a comprender el escenario en el que ocurre esta intoxicación y explorar elementos que puedan facilitar la sospecha clínica. Llamamos escenario al conjunto de circunstancias que rodean la intoxicación y que incluidos en la anamnesis o la observación directa por los equipos de salud, permiten plantear la intoxicación.

\section{Objetivo general}

Contribuir a fortalecer capacidades clínicas para sospechar y confirmar intoxicaciones por CO. El objetivo específico es describir las circunstancias que rodean estos casos, especialmente las fuentes que generan el gas y su relación con las características clínicas y demográficas.

\section{Metodología}

Es un estudio descriptivo transversal de las consultas registradas en el Centro de Información y Asesoramiento Toxicológico (CIAT) entre el $1^{\circ}$ de enero y el 31 de diciembre de 2017. Se consideró caso sospechoso todo paciente con sintomatología neurológica o digestiva de instalación aguda que ocurre en un ambiente interior en presencia de una fuente de combustión. Se incluyeron además como caso sospechoso los familiares o cohabitantes que compartieron el ambiente con la fuente de combustión identificada, independientemente de la presencia o no de síntomas. Se excluyeron los casos de sospecha de intoxicación por $\mathrm{CO}$ que estuvieron expuestos a humo de incendio. Se excluyeron los casos con noción de sobredosis de psicofármacos y alcohol asociados.

Los casos se seleccionaron del sistema de registro de las consultas del CIAT (sistema IPCS INTOX) y se analizaron manualmente los datos de las historias clínicas anonimizadas integradas a una base de datos Excel. Las variables analizadas fueron: edad, sexo, procedencia por departamento, mes del año de la consulta, latencia de la consulta, latencia entre la exposición y el análisis de $\mathrm{COHb}$, la sintomatología inicial, la ocurrencia individual o colectiva y la severidad de los casos clasificada de acuerdo a la escala de severidad de las intoxicaciones (Poison Severity Score de 


\begin{tabular}{|lcc|}
\hline \multicolumn{3}{l}{ Tabla 1. Distribucion de los casos según rangos de } \\
edad. \\
\hline Edad (años) & $N^{\circ}$ casos. FA & $F R \%$ \\
\hline 0 a 5 & 15 & 13,5 \\
6 a 14 & 22 & 19,8 \\
15 a 29 & 28 & 25,2 \\
30 a 44 & 20 & 18,0 \\
45 a 59 & 11 & 9,9 \\
Mayores de 60 & 15 & 13,5 \\
Total & 111 & $100 \%$ \\
\hline
\end{tabular}

International Programme on Chemical Safety - European Association of Poisons Centres and Clinical Toxicologists IPCS - EAPCCT). Se analizó la distribución estacional en el año, la temperatura mínima (T mín) del día en que ocurrió el caso y la fuente sospechada de emisión de CO. Los datos de T mín fueron solicitados al Instituto Nacional Uruguayo de Meteorología (INUMET). No se analizaron aspectos clínicos, paraclínicos y evolutivos de la intoxicación, ya que el objetivo de este trabajo es analizar los elementos clínicos iniciales que permitan sospechar la intoxicación. Los datos se presentaron en tablas y para interpretar estadísticamente si algunas variables estudiadas se correlacionan entre sí, se aplicó la prueba de chi cuadrado.

\section{Resultados}

En el período estudiado se registraron 111 casos de sospecha de intoxicación por $\mathrm{CO}, 60 \%$ de sexo masculino y $40 \%$ femenino. La distribución por edad mostró una media de 29 años, con un rango de 1 mes a 89 años. En la tabla 1 se presenta el número de casos y la distribución en rangos etarios.

De los 111 casos, 52 fueron identificados en el contexto de un episodio agudo colectivo. Estos casos estuvieron asociados a 22 episodios que afectaron de dos a cuatro personas. Los restantes fueron identificados como casos individuales. En el contexto de las intoxicaciones colectivas se encuentran 23/37 (62,1\%) menores de 15 años y 11/15 (73,3\%) menores de 5 años. La asociación entre edad menor de 15 años y también la edad menor de 5 años, en el contexto de intoxicación colectiva, fue significativa $(\mathrm{p}<0,05)$.

La distribución en el año se muestra en la tabla 2. Los casos de intoxicación por $\mathrm{CO}$ ocurrieron entre los meses de abril y noviembre. Considerando período invernal los meses de junio, julio y agosto, se encuentra que 67/111 $(60,3 \%)$ casos ocurrieron en este período. La frecuencia
Tabla 2. Relación de número de caso por mes y el valor medio de la temperatura mínima.

\begin{tabular}{lccc|}
\hline Mes & $T$ min media $\left({ }^{\circ} \mathrm{C}\right)$ & $N^{\circ}$ de casos & FR \% \\
\hline Abril & 12,2 & 7 & 6,3 \\
Mayo & 10,7 & 18 & 16,2 \\
Junio & 6,7 & 21 & 18,9 \\
Julio & 6,7 & 29 & 26,1 \\
Agosto & 11,3 & 17 & 15,3 \\
Setiembre & 11,4 & 12 & 10,8 \\
Octubre & 11,3 & 4 & 3,6 \\
Noviembre & 14,6 & 3 & 2,7 \\
Total & & 111 & 100 \\
\hline
\end{tabular}

disminuye a medida que se aleja de los períodos de frío. $\mathrm{El}$ análisis de la $\mathrm{T}$ mín del día de la intoxicación por $\mathrm{CO}$ varió entre $-1,5$ a $22^{\circ} \mathrm{C}$. La media de la $\mathrm{T}$ mín en los casos descritos fue de $9,3^{\circ} \mathrm{C}$.

Las fuentes de emisión de $\mathrm{CO}$ identificadas en estos casos se describen en la tabla 3. La descripción incluye el equipo y el combustible utilizado. Analizados individualmente, el equipo que ocasionó más casos fue el calefón a gas, 23/111 (20,7\%). Los aparatos utilizados para calefacción del ambiente reunidos (estufas, braseros y garrafa con accesorio) representaron 60/111 (54,0\%).

En la tabla 4 se analiza la distribución de los casos según las fuentes, observándose una clara asociación entre los equipos de calefacción y el mencionado período invernal $(p<0,01)$. Una distribución más homogénea en el año se observó cuando se trató de calefones, cocinas, generadores y otros. Los casos asociados a calefones no mostraron asociación significativa con el período invernal.

La procedencia de los casos según departamento del país se describe en la tabla 5. Se calcula la tasa según el número de habitantes. La tasa de intoxicación por $\mathrm{CO}$ cada 100.000 habitantes fue de $3,17 \pm 3,41$ para el país, y la distribución por departamento mostró que Maldonado tiene la mayor tasa (1,38/100.000 habitantes).

Con respecto al tiempo de latencia entre la exposición y la consulta se observó que 41/111 (36,9\%) demoró una hora o menos en consultar, 34/111 (30,6\%) entre una y tres horas y 31/111 (27,9\%) demoró más de tres horas. En cinco casos el tiempo de latencia fue desconocido.

La distribución de los síntomas se muestra en la tabla 6. La pérdida de conciencia transitoria se definió como una pérdida de conocimiento de comienzo abrupto, corta duración y recuperación espontánea y completa. En 
Tabla 3. Distribución de los casos según el combustible y la fuente generadora de CO identificada.

\begin{tabular}{llll}
\hline Equipo de combustión & Tipo de combustible & $F A$ & $F R \%$ \\
\hline Calefón & Gas & 23 & 20,7 \\
Estufa & Gas & 21 & 18,9 \\
Brasero y quemadores precarios & Leña & 16 & 14,4 \\
Garrafa 3 k con accesorios & Gas & 15 & 13,5 \\
Cocina & Gas & 11 & 9,9 \\
Vehículo & Combustible & 7 & 6,3 \\
Caldera & Gas & 6 & 5,4 \\
Estufa/hogar & Leña & 5 & 4,5 \\
Estufa & Kerosene & 3 & 2,7 \\
S/d & s/d & 2 & 1,8 \\
Horno & Leña & 1 & 0,9 \\
Motor de bomba & Combustible & 1 & 0,9 \\
Total $(\mathrm{n})$ & & 111 & $100 \%$ \\
\hline
\end{tabular}

este trabajo consideramos pérdida de conciencia transitoria a los casos descritos con esta terminología e incluyó los casos reportados como lipotimia o síncope.

Se obtuvieron resultados de $\mathrm{COHb}$ en $74 / 111(66,6 \%)$ casos. La media de la $\mathrm{COHb}$ fue de $14,7 \%$, con un rango de $0,3 \%$ a $62 \%$. De ellas, $41 / 74(55,4 \%) \mathrm{COHb}$ fueron mayores a $10 \%$.

La media del tiempo transcurrido entre el ingreso y la extracción de la muestra de $\mathrm{COHb}$ fue de tres horas, con un rango de 30 minutos a 48 horas. El tiempo transcurrido entre el ingreso y la dosificación de $\mathrm{COHb}$ mayor a $10 \%$ mostró, en promedio, 1 hora. En los casos con $\mathrm{COHb}$ cuyo valor fue menor a $10 \%$, el promedio de la demora fue de 4 horas. La correlación entre valores de $\mathrm{COHb}$ menor o mayor de $10 \%$, no mostró asociación significativa con el tiempo trascurrido entre el ingreso y la toma de muestra.

La clasificación por severidad (tabla 7) mostró 45/111 (40,5\%) graves, 15/111 (13,5\%) moderados, $46 / 111(41,4 \%)$ leves y $5 / 111(4,5 \%)$ de los casos fueron asintomáticos. No se encontró correlación entre la clasificación según la gravedad y el tiempo de latencia a la consulta. No hubo diferencias significativas en el porcentaje de casos severos entre niños y adultos. Se encontró una correlación significativa entre los niveles de $\mathrm{COHb}$ mayor a $20 \%$ y los casos clasificados como severos al momento de la consulta $(\mathrm{p}<0,05)$.

El análisis de la severidad entre las personas de un mismo grupo afectado (intoxicaciones colectivas) mues- tra que en 12/22 episodios de exposición los pacientes ingresan con diferentes niveles de severidad, encontrándose casos severos y leves en un mismo grupo.

En la tabla 8 se relaciona la distribución de la edad de los pacientes y la severidad de la intoxicación de acuerdo a la fuente de emisión de CO sospechada. Se halló una asociación significativa entre los casos clasificados como moderados-graves y el calefón a gas como fuente generadora de $\mathrm{CO}(\mathrm{p}<0,05)$. Esta asociación no se encontró para otras fuentes. La media de edad entre los pacientes cuya fuente de exposición fue un calefón a gas, fue de 11,9 años.

\section{Discusión y comentarios}

Se incluyeron los casos de sospecha de intoxicación por CO que fueron consultados al CIAT desde los centros de salud de todo el país y del Sistema Nacional Integrado de Salud (SNIS). La frecuencia de intoxicación por $\mathrm{CO}$ en este estudio muestra una tasa más baja que la de Estados Unidos, España y Argentina. La frecuencia encontrada se mantiene en los últimos diez años, ya que en 2009 se reportaron un promedio de 120 casos anuales ${ }^{(14)}$. Se estima una incidencia de consultas de emergencia por intoxicación por $\mathrm{CO}$ en Estados Unidos de 16 casos por 100.000 habitantes y dos tercios corresponden a exposiciones no intencionales que ocurren en ambientes interiores ${ }^{(15)}$. En Argentina, la tasa promedio de notificaciones es de 8 cada 100.000 habitantes ${ }^{(16)}$. 
Tabla 4. Distribución de fuente de CO por mes.

\begin{tabular}{lcccc}
\hline Mes & Calefacción & Calefón & Otros & Total \\
\hline Abril & 4 & 1 & 2 & 7 \\
Mayo & 6 & 5 & 7 & 18 \\
Junio & 12 & 3 & 1 & 21 \\
Julio & 23 & 5 & 5 & 29 \\
Agosto & 8 & 4 & 4 & 12 \\
Setiembre & 5 & 3 & 0 & 4 \\
Octubre & 2 & 2 & 3 & 3 \\
Noviembre & 0 & 0 & 28 & 111 \\
Total & 60 & 23 & & \\
\hline
\end{tabular}

Tabla 5. Prevalencia de intoxicación por CO según departamento.

\begin{tabular}{|c|c|c|c|}
\hline Departamento & $F A$ & Población (INE 2017) & Tasa c/100.000/hab \\
\hline Montevideo & 48 & 1.381 .228 & 3,47 \\
\hline Maldonado & 26 & 187.576 & 13,86 \\
\hline Canelones & 13 & 581.532 & 4,47 \\
\hline San José & 6 & 115.584 & 5,19 \\
\hline Colonia & 4 & 130.008 & 3,07 \\
\hline Paysandú & 3 & 119.094 & 2,52 \\
\hline Flores & 2 & 26.504 & 7,55 \\
\hline Florida & 2 & 69.312 & 2,88 \\
\hline Rivera & 2 & 108.319 & 1,85 \\
\hline Durazno & 1 & 58.996 & 1,7 \\
\hline Lavalleja & 1 & 59.161 & 1,69 \\
\hline Río Negro & 1 & 57.644 & 1,73 \\
\hline Rocha & 1 & 73.999 & 1,35 \\
\hline Tacuarembó & 1 & 93.039 & 1,07 \\
\hline Artigas & 0 & 74.810 & 0 \\
\hline Cerro Largo & 0 & 89.557 & 0 \\
\hline Salto & 0 & 132.294 & 0 \\
\hline Soriano & 0 & 84.032 & 0 \\
\hline Treinta y Tres & 0 & 50.516 & 0 \\
\hline Uruguay & 111 & 3.493 .205 & 3,17 \\
\hline
\end{tabular}




\begin{tabular}{|lcc|}
\hline Tabla 6. Frecuencia de síntomas. & & \\
\hline Síntomas & $F A$ & $F R \%$ \\
\hline Cefalea & 49 & 24,6 \\
Pérdida conocimiento transitoria & 40 & 20,1 \\
Vómitos & 26 & 13,1 \\
Náuseas & 26 & 13,1 \\
Mareos & 25 & 12,5 \\
Debilidad muscular & 9 & 4,5 \\
Disnea & 6 & 3,0 \\
Hiporreactividad & 5 & 2,5 \\
Dolor torácico & 4 & 2,0 \\
Convulsión & 4 & 2,0 \\
Coma & 2 & 1,0 \\
Hemiparesia & 1 & 0,5 \\
Confusión & 1 & 0,5 \\
Acúfenos & 199 & 0,5 \\
Total & & 100 \\
\hline & & \\
\hline
\end{tabular}

En nuestro estudio, la tasa promedio es mucho menor y aun los departamentos con tasas más elevadas están en valores muy bajos respecto a las cifras referidas en otras regiones. Se estima que estas cifras puedan reflejar un subdiagnóstico o un subregistro. Varios autores señalan un elevado número de intoxicaciones ocultas por CO en la mayoría de las poblaciones, sugiriendo cifras mayores a $30 \%$ y $40 \%$ de intoxicaciones inadverti$\operatorname{das}^{(17,18)}$.

Estos casos no diagnosticados permiten la persistencia del riesgo, la exposición crónica y eventualmente nuevos episodios agudos. Los casos no diagnosticados no reciben el tratamiento antidótico con $\mathrm{O}_{2}$, ya sea nor- mobárico o hiperbárico. Este último es un tratamiento de alta especificidad, con evidencia creciente sobre su eficacia, para el tratamiento del episodio agudo y para evitar o minimizar secuelas ${ }^{(19)}$.

Entre los elementos clínicos más orientadores de intoxicación por $\mathrm{CO}$ se encuentra el episodio que afecta simultáneamente a dos o más personas que comparten un espacio interior. En todos los casos hay consenso en la búsqueda activa de otras personas del entorno del paciente y la dosificación de la $\mathrm{COHb}$, aunque estén asintomáticos ${ }^{(16)}$.

Los resultados encuentran que casi la mitad de los casos provienen de una exposición colectiva. Sin embargo, se destacan casos que cursan aislados, por lo que es necesario analizar otros elementos clínicos y del escenario que permitan orientar la sospecha diagnóstica. La asociación significativa entre intoxicación por $\mathrm{CO}$ en niños y su identificación dentro de un episodio de afectación colectiva señala la posibilidad de que casos aislados en niños puedan estar pasando desapercibidos si no hay una búsqueda activa de los casos. Los resultados de este trabajo no muestran una correlación entre severidad y edad de los pacientes. Por otra parte, la severidad de las personas afectadas en un mismo grupo fue muy variable, tal como observan otros autores ${ }^{(20)}$. Se identifican casos severos con cohabitantes asintomáticos y, como fue descrito, varios casos asintomáticos buscados activamente tenían valores de $\mathrm{COHb}$ por encima de $10 \%$. Puede estimarse que las diferencias sean debidas a tiempos de permanencia diferentes o localización más o menos cerca de la fuente de generación de $\mathrm{CO}$, o su permanencia en un sector de la habitación con mejor ventilación.

Se reconoce ampliamente que la mayor causa de intoxicación por $\mathrm{CO}$ es el equipo a combustión incorrectamente instalado o mal mantenido, situado en ambientes interiores con escasa o nula ventilación ${ }^{(12,13)}$.

El CO se genera por la combustión incompleta, que es la permanencia del fuego utilizando una menor cantidad de $\mathrm{O}_{2}$. La combustión incompleta (o mala combustión) forma más $\mathrm{CO}$ que dióxido de carbono $\left(\mathrm{CO}_{2}\right)$. Esto ocurre cuando no hay buen recambio de aire, ya que el

Tabla 7. Relación de severidad y valor de $\mathrm{COHb}$.

\begin{tabular}{lccccc}
\hline Severidad & $<$ a $10 \%$ & $>10$ a $19 \%$ & $>20 \%$ & No se realizó & Total \\
\hline Asintomático & 0 & 3 & 1 & 1 & 5 \\
Leve & 16 & 4 & 3 & 23 & 46 \\
Moderado & 5 & 3 & 3 & 4 & 15 \\
Severo & 12 & 9 & 15 & 9 & 45 \\
Total & 33 & 19 & 22 & 37 & 111 \\
\hline
\end{tabular}


Tabla 8. Distribución de la edad de los pacientes y la severidad de la intoxicación según fuente.

\begin{tabular}{lccc}
\hline Fuente & $N^{0}$ de casos & Edad media (rango) & Severidad Mod-Grave \\
\hline Calefón a gas & 23 & 13 años (2-29) & $17 / 23$ \\
Estufa a gas & 21 & 34 años (1-78) & $9 / 21$ \\
Brasero y quemadores precarios & 16 & 29 años (2-70) & $8 / 16$ \\
Garrafa 3 k con accesorios gas & 15 & 29 años (2-83) & $9 / 15$ \\
Cocina gas & 11 & 43 años (1-89) & $5 / 11$ \\
\hline
\end{tabular}

$\mathrm{O}_{2}$ es la sustancia comburente. El mal funcionamiento de los equipos debido a obstrucción, suciedad o deterioro, las chimeneas obstruidas o solo la falta de recambio de aire en los ambientes interiores puede ocasionar la mayor producción y acumulación de $\mathrm{CO}$ en el espacio donde las personas permanecen ${ }^{(17)}$.

Las fuentes descritas en este estudio se repiten en reportes de otros países, más allá de sus diferentes características de tamaño o modelo. Están asociadas a la calefacción de ambientes interiores, procesos de cocción de alimentos, calentamiento de agua y generación de energía. La mayoría de los casos provienen del hogar, pero también se identificaron casos asociados a motores portátiles en recintos de trabajo, generadores de energía, así como fugas de CO al interior de los vehículos en marcha ${ }^{(6,17)}$.

Las fuentes de calefacción utilizadas en Uruguay son en un $96 \%$ provenientes de la combustión (estufa o quematuti a leña $54 \%$ y estufas a supergás $42 \%$ ). La fuente de cocción de alimentos es en $90 \%$ la cocina y horno a supergás ${ }^{(21)}$. Considerando que en 2013 solo el $1 \%$ de los uruguayos tenía calefón a gas, llama la atención que la fuente de intoxicación más frecuente sea ésta en 2017. Esto sugiere un aumento de su uso o la posibilidad de que hayan sido instalados en condiciones inseguras, sobre todo aquellos que deben instalarse en lugares ventilados (terrazas, patios, etc.). La asociación entre la fuente calefón y la severidad de los casos de intoxicación puede ser explicada por el altísimo riesgo de este escenario, ya que son espacios pequeños y cerrados. Las cocinas representaron escenarios de riesgo de menor frecuencia. Una explicación posible es que la cocción de alimentos es un proceso de duración acotada en relación con los calefactores o estufas a combustión. Si así fuera, cabría esperar intoxicaciones cuando se realizan cocciones de larga duración en espacios cerrados. Mc Cann y colaboradores describen que además del mal funcionamiento o estado de las cocinas, la generación de $\mathrm{CO}$ puede ser mayor cuando se colocan ollas de diámetro superior al de la hornalla, lo que causa una disminución de la captación de aire para la combustión ${ }^{(12)}$.
La asociación de los casos de intoxicación con el período invernal es lo esperable, ya que el aumento de la frecuencia de intoxicaciones por $\mathrm{CO}$ ocurre mayormente debido a la presencia de combustión incompleta de fuentes de calefacción ${ }^{(6,14,17)}$.

La media de la T mín ambiental en las fechas donde ocurrieron los casos estudiados permite predecir que cuando la $\mathrm{T}$ mín está por debajo de $10^{\circ} \mathrm{C}$ habrá un aumento de los casos de intoxicación por CO. La mayor frecuencia de casos en Uruguay en julio es coincidente con el promedio de T mín en este mes, que en los últimos años ronda los $7^{\circ} \mathrm{C}$ y es el más bajo del año ${ }^{(22)}$.

En 2017, todo el país presentó valores de temperatura por encima del promedio de los años anteriores, con máximos desvíos positivos en la región sur-sureste, por lo que cabe plantearse que en años aún más fríos pueda haber un aumento del número de casos ${ }^{(23)}$.

En este estudio los síntomas más frecuentes que los pacientes presentaron al ingreso fueron: cefaleas, pérdida transitoria de conciencia, vómitos, náuseas y mareos (tabla 6). La presentación clínica de la intoxicación ha sido ampliamente descrita en la literatura y los resultados obtenidos son coincidentes con lo reportado. La cefalea ha sido descrita como el síntoma más común de intoxicación por $\mathrm{CO}^{(11,13,15-17)}$. Sin embargo, las características clínicas de esta cefalea han sido poco estudiadas. Algunos autores plantean que el tipo, localización e intensidad es muy variable, aunque es frecuente la localización frontal. El diagnóstico diferencial más frecuente es la migraña y la cefalea tensional ${ }^{(24)}$. En las intoxicaciones por $\mathrm{CO}$, la cefalea se asocia a niveles de $\mathrm{COHb}$ mayor de $10 \%$, lo que sugiere que es un síntoma clave para el diagnóstico temprano de la intoxicación ${ }^{(11)}$.

Los casos que consultan por malestar general, decaimiento, debilidad muscular, náuseas y dificultad respiratoria pueden ser interpretados como gripes estacionales, aunque la intoxicación por $\mathrm{CO}$ no causa fiebre. En casos de intoxicaciones colectivas que se presentan con náuseas y vómitos, el diagnóstico inicial tiende a ser el de intoxicación alimentaria. La exposición a mayor do- 
sis durante mayor tiempo ocasiona síntomas neurológicos como ataxia, vértigos, intensa debilidad muscular, alteración del nivel de conciencia y sobre todo convulsiones en niños ${ }^{(14)}$. La pérdida de conciencia de corta duración, que revierte al poco tiempo de que ocurra la ventilación del lugar o el traslado a un centro asistencial, es un síntoma característico de esta intoxicación. La pérdida de conciencia es el síntoma de mayor valor predictivo de complicaciones y secuelas ${ }^{(25)}$.

La inespecificidad de los síntomas de la intoxicación ha llevado a investigar prospectivamente la prevalencia de la intoxicación por $\mathrm{CO}$ de pacientes que consultan en emergencias hospitalarias por cefaleas, con síntomas gripales, dolor precordial y convulsiones. Los resultados de estos estudios mostraron que $7,5 \%$ de los casos con cefaleas, $6,3 \%$ con síndromes gripales, $4,3 \%$ con dolor precordial y $3,3 \%$ con convulsiones tenían valores de $\mathrm{COHb}$ por encima de $5 \%{ }^{(11)}$.

Entre los casos de este estudio, no se identificó el color rojo de la piel, considerado característico en estudios forenses, ni el enrojecimiento ocular por vasodilatación que se describe en casos de intoxicación moderada y severa $^{(4)}$.

La dosificación de $\mathrm{COHb}$ en sangre confirma la presencia de CO unido a la hemoglobina. Será el valor máximo alcanzado y su persistencia lo que explique la sintomatología y posteriormente las secuelas. Los valores elevados en sangre confirman la exposición, pero los valores bajos no la descartan. La medida de la $\mathrm{COHb}$ es una fotografía que pierde representatividad a medida que trascurre el tiempo ${ }^{(11,13)}$. Otros estudios complementarios como la gasometría, el nivel de lactato, el ECG, el tropo test, y la tomografía o resonancia nuclear magnética de cráneo son evaluados para establecer la gravedad, pero en sí mismos no son un aporte específico al diagnóstico etiológico ${ }^{(25)}$.

El CO es 200 veces más afín a la hemoglobina que el $\mathrm{O}_{2}$ y la vida media de la unión CO-hemoglobina respirando al aire es de unas cuatro horas, y si el paciente recibe oxigenoterapia, la vida media cae a dos horas ${ }^{(14)}$. Transcurrido cierto tiempo o si se administra oxigenoterapia previa a la extracción de la muestra para dosificación de $\mathrm{COHb}$, esta pierde valor para evaluar la intensidad de la exposición porque habrá disminuido ${ }^{(20)}$.

En nuestra serie los niveles promedio de $\mathrm{COHb}$ observados fueron promediamente más bajos en quienes consultaron más tardíamente, aunque la correlación observada con el tiempo transcurrido no fue significativa.

Tampoco se encontró correlación clínica-analítica estricta entre $\mathrm{COHb}$ y la severidad al momento de toma de la muestra, lo que es esperable a la luz de lo reportado por otros autores ${ }^{(17,25)}$. La COHb tiene valor diagnóstico cuando está elevada, pero la evidencia muestra que por sí sola no predice complicaciones ni secuelas a largo plazo $^{(26)}$. García y colaboradores encuentran una asociación entre síntomas o signos de daño cardiovascular con niveles mayores a $20 \%{ }^{(6)}$. Los resultados observados están en sintonía con este reporte, ya que los casos con $\mathrm{COHb}$ mayor a $20 \%$ se asocian significativamente con los casos clasificados como moderados y severos ${ }^{(6)}$.

La distribución geográfica por departamento muestra a Maldonado con la mayor tasa de intoxicaciones y que la fuente de combustión involucrada fue mayoritariamente los calefones a gas. Este resultado conlleva la necesidad de nuevas investigaciones que permitan comprender si hay características particulares de los calefones comercializados o factores relacionados con el tipo y lugar de instalación. Llama la atención la ausencia de casos procedentes de Soriano, ya que en este departamento es donde se registraron las T mín más bajas del país, según INUMET. Sería necesario investigar cuáles pueden ser los factores que explican esta ausencia de casos.

Jhones y colaboradores plantean la importancia de conocer las características de los equipos, su forma de uso e instalación y su mantenimiento. Son principios básicos para la prevención de la intoxicación, no solo el mantenimiento de los equipos, sino también el aseguramiento de recambio de aire. Los monitores ambientales tienen diferente calidad y efectividad. Se recomiendan en espacios de uso frecuente y prolongado de aparatos a combustión. Se destaca igualmente la necesidad de incrementar la sospecha clínica temprana como disparador para diagnosticar, investigar y resolver este riesgo ambiental $^{(27)}$.

Keles y colaboradores describen casos de intoxicación aguda que eran de hecho pacientes con exposición crónica no identificada previamente y plantean que la exposición aguda debe considerarse un indicador de exposición crónica o repetida ${ }^{(28)}$.

La toxicidad del $\mathrm{CO}$ es función de la concentración en el aire y el tiempo de exposición. El nivel promedio de $\mathrm{CO}$, sin fuentes de combustión, depende del valor que hay en el aire exterior y se reporta entre 1 y 4 ppm. Las intoxicaciones agudas se asocian frecuentemente a valores de $\mathrm{CO}$ en aire mayor de $100 \mathrm{ppm}$. Valores de 200 ppm en aire causan cefaleas si transcurren dos horas de exposición y valores de $400 \mathrm{ppm}$ causan el mismo síntoma en una hora. Valores mayores de 100 ppm se han medido en ambientes interiores asociados a fuentes de combustión ${ }^{(29)}$.

\section{Conclusiones}

La intoxicación por $\mathrm{CO}$ es una patología de origen ambiental, frecuente en el período invernal, asociada a temperaturas bajas y al encierro en ambientes interio- 
res. El inicio del período invernal y los períodos con $\mathrm{T}$ mín menor a $10{ }^{\circ} \mathrm{C}$ plantean un alerta sobre el aumento de casos.

Los resultados de este estudio plantean la necesidad de incorporar preguntas sobre el escenario en el que se encuentran los pacientes en el momento del inicio de los síntomas o dónde son hallados. Preguntar sistemáticamente la presencia de fuentes de combustión en ambientes poco ventilados permitirá sospechar y en ese caso confirmar tempranamente la intoxicación. Los equipamientos de combustión a gas o a leña son en conjunto los más frecuentes, pero se destacan individualmente los calefones y las estufas a gas. El escenario de intoxicación asociado a un calefón a gas se presenta como el de más alto riesgo, probablemente porque el $\mathrm{CO}$ alcance concentraciones elevadas muy rápidamente en un ambiente pequeño, como lo es habitualmente un baño.

Las cefaleas se presentan como el síntoma inicial más frecuente, por lo que debe considerarse un síntoma clave en el escenario de consulta invernal. La pérdida transitoria de conciencia, siguiente síntoma en frecuencia, es un síntoma característico de esta intoxicación y también es predictor de severidad y secuelas posteriores. Su presencia, en el marco de un escenario sospechoso, es uno de los criterios clínicos de indicación de oxigenoterapia en cámara hiperbárica. La dosificación de $\mathrm{COHb}$ es el examen de confirmación diagnóstica. Su valor elevado confirma exposición reciente, aunque un valor medido tardíamente no permite descartarla.

Los resultados plantean que también en esta casuística puede haber un subdiagnóstico o un subregistro del CIAT. Si bien los episodios colectivos se sospechan con mayor facilidad, hay una importante proporción de casos individuales. Se plantea, en el caso de los niños, que el diagnóstico de casos individuales pueda pasar desapercibido en mayor proporción que en los adultos, ya que los casos pediátricos hallados se asociaron significativamente con intoxicaciones colectivas.

Los datos observados contribuyen a incrementar la capacidad de sospecha de esta intoxicación, pero son necesarios más estudios que profundicen en los aspectos clínicos, analíticos y ambientales, para prevenir la toxicidad del CO, promover el uso de fuentes de combustión ambientalmente seguras y detectar precozmente la intoxicación.

\section{Agradecimiento}

Al Instituto Uruguayo de Meteorología (INUMET).

\section{Summary}

Carbon monoxide accumulates in closed environments when there is unnoticed combustion. Signs of poisoning are non-specific, and thus circumstances around poisoning may constitute a diagnostic guide.

Objective: to strengthen the clinical capacities to suspect and confirm carbon monoxide poisoning.

Method: descriptive, transversal study of suspicious cases (patients with neurological or digestive symptoms for which an indoor combustion source is identified) who consulted at the Poisoning Center in 2017.

Results: 111 cases were included in the study, 52 of which occurred in 22 episodes of collective exposure. 37 patients were under 15 years old and the percentage of children identified in collective poisoning (23/37) was significantly greater than the one representing adults. 67 cases occurred in winter, minimum average temperature being $9.3^{\circ} \mathrm{C}$. The combustion equipment most frequently involved in poisoning events were gas-fired water heaters in the first place, followed by gas stoves. The most frequent initial symptom was headache. Average COHb was $14.7 \%$. Severe cases (45/111) were significantly corelated to carbon monoxide exposure from gas-fired water heaters.

Conclusions: the poisoning rate is low when compared to countries in a similar latitude, what suggests the possibility of under-diagnosis. Minimum environment temperature under $10^{\circ} \mathrm{C}$, staying in spaces with combustion-based heating and the use of gas-fired water heaters were the typical poisoning scenarios. Headache is a key symptom to search for exposure. The diagnostic suspicion may be lower when it involves individual cases, mainly in children.

\section{Resumo}

O monóxido de carbono (CO) se acumula em ambientes fechados quando há combustão e não é perceptível. Os sintomas de envenenamento são inespecíficos, portanto, as circunstâncias em que ocorre o envenenamento podem orientar o diagnóstico.

Objetivo: fortalecer as capacidades clínicas para suspeitar e confirmar envenenamento por monóxido de carbono.

Método: estudo descritivo transversal de casos suspeitos (pacientes com sintomas neurológicos ou digestivos em que uma fonte de combustão é identificada em ambiente interno) consultados no CIAT em 2017.

Resultados: foram identificados 111 casos, dos quais 52 ocorreram em 22 episódios de exposição em grupo. Trinta e sete eram menores de 15 anos sendo que a porcentagem de crianças que foram identificadas em intoxicações coletivas (23/37) é significativamente maior do que em adultos. No "período de inverno", com temperatura média mínima de $9,3^{\circ} \mathrm{C}$, ocorreram $67 \mathrm{ca}$ sos. Os equipamentos de combustão mais frequente- 
mente envolvido foram aquecedores de água a gás, seguidos de fogões a gás. O sintoma inicial mais frequente foi cefaleia. O COHb médio foi de $14,7 \%$. Os casos graves $(45 / 111)$ foram significativamente correlacionados com a exposição ao $\mathrm{CO}$ de um aquecedor de água a gás.

Conclusões: o índice de intoxicações é baixo quando comparado a países de latitude semelhante, o que indica a possibilidade de subdiagnóstico. A temperatura ambiente mínima abaixo de $10^{\circ} \mathrm{C}$, a permanência em ambientes com aquecimento a combustão e o uso de aquecedores a gás foram os cenários típicos de intoxicação. A cefaleia é um sintoma chave para investigar a exposição. A suspeita diagnóstica pode ser menor quando se trata de casos individuais, principalmente em crianças.

\section{Bibliografía}

1. Ng PC, Long B, Koyfman A. Clinical chameleons: an emergency medicine focused review of carbon monoxide poisoning. Intern Emerg Med 2018; 13(2):223-9.

2. Nogué S, Dueñas A. Monóxido de carbono: un homicida invisible y silencioso. Med Clin (Barc) 2005; 124(8):300-1

3. Wright J. Chronic and occult carbon monoxide poisoning: we don't know what we're missing. Emerg Med J 2002; 19(5):386-90.

4. Ashcroft J, Fraser E, Krishnamoorthy S, Westwood-Ruttledge S. Carbon monoxide poisoning. BMJ 2019; 365:12299.

5. Buchelli H, Fernández R, Rubinos G, Martínez C, Rodríguez F, Pere Casan C. Niveles elevados de carboxihemoglobina: fuentes de exposición a monóxido de carbono. Arch Bronconeumol 2014; 50(11): 465-8.

6. García S. Guía de prevención, diagnóstico, tratamiento y vigilancia epidemiológica de las intoxicaciones por monóxido de carbono. $2^{\mathrm{a}}$ ed. Buenos Aires: Ministerio de Salud de la Nación. Programa Nacional de Prevención y Control de las Intoxicaciones, 2014.

7. Satran D, Henry CR, Adkinson C, Nicholson CI, Bracha Y, Henry TD. Cardiovascular manifestations of moderate to severe carbon monoxide poisoning. J Am Coll Cardiol 2005; 45:1513-6.

8. Karaman S, Coskun A. Risk of late appearance of acute myocardial infarction after carbon monoxide (CO) intoxication. Rev Med Chile 2019; 147(9):1128-35.

9. Yurtseven S, Arslan A, Eryigit U, Gunaydin M, Tatli O, Ozsahin F, et al. Analysis of patients presenting to the emergency department with carbon monoxide intoxication. Turk J Emerg Med 2015; 15(4):159-62.

10. Weaver LK, Valentine KJ, Hopkins RO. Carbon monoxide poisoning: risk factors for cognitive sequelae and the role of hyperbaric oxygen. Am J Respir Crit Care Med 2007; 176(5):491-7.

11. Clarke S, Keshishian C, Murray V, Kafatos G, Ruggles R, Coultrip E. Screening for carbon monoxide exposure in se- lected patient groups attending rural and urban emergency departments in England: a prospective observational study. BMJ Open 2012; 2(6):e000877.

12. McCann L, Close R, Staines L, Weaver M, Cutter M, Leonardi G. Indoor carbon monoxide: a case study in England for detection and interventions to reduce population exposure. J Environ Public Health 2013:735952.

13. Oliu G, Nogué S, Miró Ó. Intoxicación por monóxido de carbono: claves fisiopatológicas para un buen tratamiento. Emergencias 2010; 22: 451-9.

14. Pose D, Negrin A, Laborde A. Intoxicaciones por monóxido de carbono. En: Leonardis D, Sehabiague G, Prego J, Bello O. Pediatría, urgencias y emergencias. Montevideo: Bibliomédica, 2009:1081-8.

15. Rose J, Wang L, Xu Q, McTiernan C, Shiva S, Tejero J, et al. Carbon monoxide poisoning: pathogenesis, management, and future directions of therapy. Am J Respir Crit Care Med 2017; 195(5):596-606.

16. Díaz M, Crapanzano G, Cabrerizo S, Aichele C, Deurtiaga $\mathbf{A}$, Vallejos $\mathbf{Y}$. Intoxicación masiva con monóxido de carbono: puesta al día a partir de un caso. Arch Argent Pediatr 2017; 115(1):76-81.

17. Donati S, Gainnier M, Chibane-Donati O. Intoxicación por monóxido de carbono. EMC-Anest-Reanim 2005; 31(2):1-17.

18. Vázquez M, Álvarez C, Cruz A, López M. Intoxicaciones inadvertidas por monóxido de carbono: una epidemia oculta. Rev Toxicol 2015; 32:98-101.

19. Lin CH, Su WH, Chen YC, Feng PH, Shen WC, Ong JR. Treatment with normobaric or hyperbaric oxygen and its effect on neuropsychometric dysfunction after carbon monoxide poisoning: a systematic review and meta-analysis of randomized controlled trials. Medicine (Baltimore) 2018; 97(39):e12456.

20. Sethuraman KN, Dougla TM, Bostick BB, Comer AC, Myers B, Rosenthal RE. Clinical characteristics of pediatric patients with carbon monoxide poisoning. Pediatr Emerg Care 2017; 36(4):178-81.

21. Uruguay. Ministerio de Industria, Energía y Minería. Dirección Nacional de Energía. Características del sector residencial, datos 2013. Encuesta de consumo. Planificación, estadística y balance. Disponible en: https://www.miem. gub.uy/energia/encuesta-sobre-consumo-y-usos-de-la-energia-en-el-sector-residencial-datos-2013 [Consulta: 25 junio 2020].

22. Instituto Uruguayo de Meteorología. El invierno: año 2015. Montevideo, 2015 Disponible en: https://www.inumet.gub.uy/reportes/dcd/invierno.pdf [Consulta: 21 junio 2020].

23. Instituto Uruguayo de Meteorología. Tendencias Climáticas Agosto-Setiembre-Octubre 2017.Montevideo, 2017. Disponible en: https://www.inumet.gub.uy/index.php/clima/tendencias-climaticas/tendencias-climaticas-agosto-setiembre-octubre-2017 [Consulta: 21 junio 2020]. 
24. Hampson NB, Hampson LA. Characteristics of headache associated with acute carbon monoxide poisoning. Headache 2002; 42(3):220-3.

25. Chiew AL, Buckley NA. Carbon monoxide poisoning in the 21st century. Crit Care 2014; 18:221.

26. Köthe L, Radke J. Carboxyhemoglobin concentration in carbon monoxide poisoning. Critical appraisal of the predictive value. Anaesthesist 2010; 59(6):529-34.

27. Jones S, Horton A, Brunt H, Shankar G. Preventing carbon monoxide poisoning. BMJ 2019; 366:14613.
28. Keles A, Demircan A, Kurtoglu G. Carbon monoxide poisoning: how many patients do we miss? Eur J Emerg Med 2008, 15:154-7.

29. Penney D, Benignus V, Kephalopoulos S, Kotzias D, Kleinman M, Verrier A. Carbon monoxide. En: WHO Guidelines for indoor air quality. Selected pollutants. Geneva: WHO, 2010.

\section{Contribución de autoras}

Las autoras hemos trabajado igualmente en las etapas de concepción, diseño, ejecución, redacción, realización de conclusiones y revisión crítica.

Amalia Laborde García, ORCID 0000-0001-5793-1226

María Noel Tortorella, ORCID 0000-0001-9400-3648 